\title{
Timing Analysis with INTEGRAL: Comparing Different Reconstruction Algorithms
}

\author{
V. Grinberg, I. Kreykenbohm, F. Fürst, J. Wilms, K. Pottschmidt, M. Cadolle Bel, \\ J. Rodriguez, D. M. Marcu, S. Suchy, A. Markowitz, M. A. Nowak
}

\begin{abstract}
INTEGRAL is one of the few instruments capable of detecting X-rays above $20 \mathrm{keV}$. It is therefore in principle well suited for studying X-ray variability in this regime. Because INTEGRAL uses coded mask instruments for imaging, the reconstruction of light curves of X-ray sources is highly non-trivial. We present results from a comparison of two commonly employed algorithms, which primarily measure flux from mask deconvolution (ii_lc_extract) and from calculating the pixel illuminated fraction (ii_light). Both methods agree well for timescales above about $10 \mathrm{~s}$, the highest time resolution for which image reconstruction is possible. For higher time resolution, ii_light produces meaningful results, although the overall variance of the lightcurves is not preserved.
\end{abstract}

Keywords: timing analysis, instrumentation and methods, lightcurves, data extraction.

\section{Introduction}

The INTEGRAL satellite is one of the few instruments designed for the detection of X-rays above $20 \mathrm{keV}$ with a good time resolution. It offers a unique opportunity for timing studies in this regime, though the exact analysis at high time resolution remains a challenge. In coded mask instruments like the IBIS telescope aboard the INTEGRAL the source radiation is modulated by a mask. Each source will cast a shadow image (shadowgram) - the combined shadowgram is recorded in the detector plane. To obtain the original image the detected flux distribution has to be deconvolved in an analytically and computationally non-trivial process which is highly CPUintensive.

For the reconstruction of lightcurves two algorithms are commonly employed: ii_lc_extract deconvolves shadowgrams for each time and energy bin, where the lightcurve is extracted. ii_light calculates the lightcurves primarily from the pixel illumi-

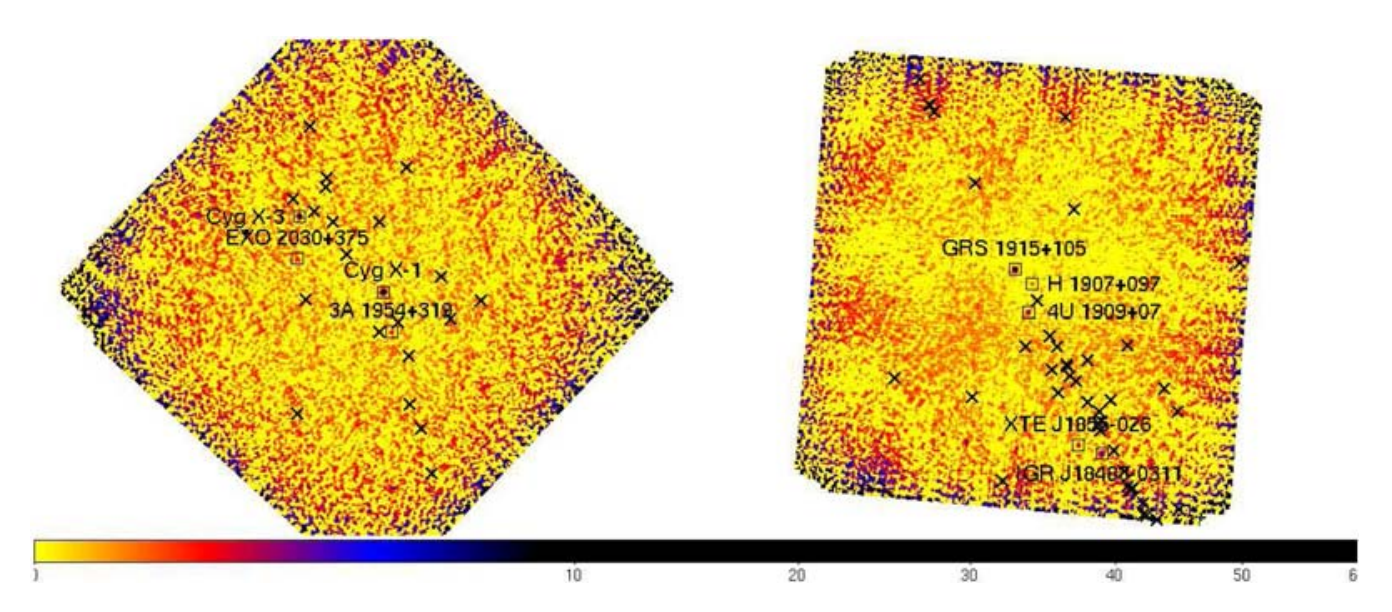

Fig. 1: Intensity mosaics of the fields of Cyg X-1 and GRS $1915+105$ in the $20-40 \mathrm{keV}$ band nated fraction (PIF, number between 0 and 1 for a given source expressing the degree of illumination of a detector pixel). Both are included in the INTEGRAL Off-line Scientific Analysis (OSA) software package.

In the following we compare the two extraction mechanisms and discuss their advantages and shortcomings (Sec. 2), and then assess the suitability of ii_light for high time resolution analysis (Sec. 3). A short summary of the results and the implications for further timing analysis with INTEGRAL are given in Sec. 4.

\section{Comparison between different lightcurve extraction algorithms}

To reduce the influence of the selected field on the results of the lightcurve extraction, we perform the comparison on two different fields. Figure 1 shows a comparison of significance mosaics obtained dur-

33 

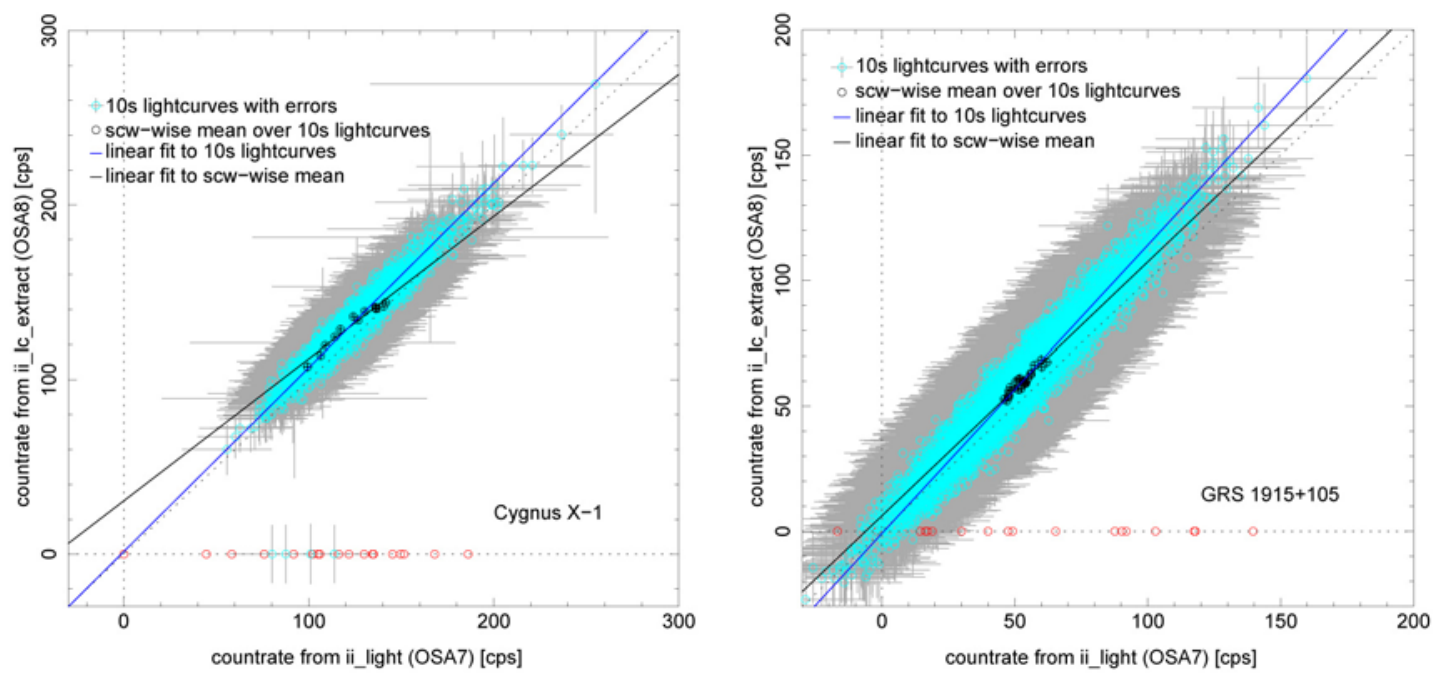

Fig. 2: Scatterplots for countrates obtained with ii_lc_extract and ii_light

ing the Cyg X-1 and GRS 1915+105 INTEGRAL key programme in the $20-40 \mathrm{keV}$ energy band with the source in the fully coded field of view (FOV), i.e. to a maximum pointing offset of $4.5^{\circ}$, from 15 science windows (ScWs) from revolution 628 and $26 \mathrm{ScWs}$ from revolution 852, respectively. Cyg X-1 (countrate $~$ $100 \mathrm{cps})$ is significantly brighter than GRS $1915+105$ ( $\sim 40 \mathrm{cps})$. While both fields are comparable regarding the sources taken into account for our extractions (named boxes, $\sigma_{\text {detection }} \geq 6$, cps $\sim 0.5-4.5$ ), the field of GRS $1915+105$ is crowded with $\sim 35$ weak sources (marked with $\times, 1 \leq \sigma_{\text {detection }}<6$ ), while the field of Cyg X-1 shows only $\sim 20$ of them.

Figure 2 shows the correlation between the results obtained with ii_light (OSA 7 version) and ii_lc_extract (OSA 8 version) for sources in the fully coded field of view in the $18-50 \mathrm{keV}$ and 20-50 keV band for Cyg X-1 and GRS 1915+105 (revolutions and ScWs as for mosaic images), respectively. Note that the ii_light algorithm was not included into OSA 8 release; we therefore used the newest version of both algorithms available at the time of writing. At a $10 \mathrm{~s}$ time resolution ii_lc_extract fails to detect the respective sources in several timebins, resulting in datapoints with zero countrate and error (red circles), which are excluded from our analysis. Negative countrate values are an artifact of the background extraction and common for X-ray lightcurves. ii_lc_extract does not allow for a much higher time resolution than $10 \mathrm{~s}$. ii_light systematically underestimates the countrate. The $10 \mathrm{~s}$ lightcurves (cyan circles) are however well linearly correlated with a bestfit slope of $1.05 \pm 0.01$ for $\mathrm{Cyg} X-1$ and $1.15 \pm 0.01$ for GRS $1915+105$. Fits to individual ScW-averaged countrates (black circles) in both cases show a different linear correlation with a lower slope and a sig- nificant offset. Given the good correlation for the non-averaged lightcurves and the fact, that the average datapoints lie well on the $10 \mathrm{~s}$ lightcurve fits, we are inclined to attribute this to the low number of ScWs analysed. More data covering a greater range in countrates will shed light on this issue.

The performance of ii_light on all available INTEGRAL Crab data was analysed by [2], who found that $i i_{-}$light underestimates the countrates by about $5 \%$, consistent with our results. We attribute the different ratios of ii_light and ii_lc_extract results for Cyg X-1 and GRS 1915+105 to the differences in the fields: in a more crowded field like the one of GRS $1915+105$, a signal is more likely to be assigned to the wrong source.

\section{High Time Resolution with ii_light}

For the following section we use all ScWs from revolution 628 where Cyg X-1 is in the fully or partially coded FOV, i.e., science windows with a pointing offset of up to $\sim 15^{\circ}$.

Histograms (width $1 \mathrm{cps}$ ) of the ii_light lightcurves for Cyg X-1 (Fig. 3) and Gaussian fits to them show that though the scatter increases with the time resolution, the routine produces meaningful results. The centers of the Gaussians fit components (dashed lines) are well consistent with each other. The FWHM of the Gaussians increases by a factor of $\sim 3$ for one order of magnitude increase in time resolution, consistent with the decreasing SNR. The deviations from the Gaussian shape are explained by the high intrinsic variability of the source of $>25 \%$ over the 3 days of the INTEGRAL revolution 628 , as seen in Fig. 4. Note also that Fig. 4 supports the find- 


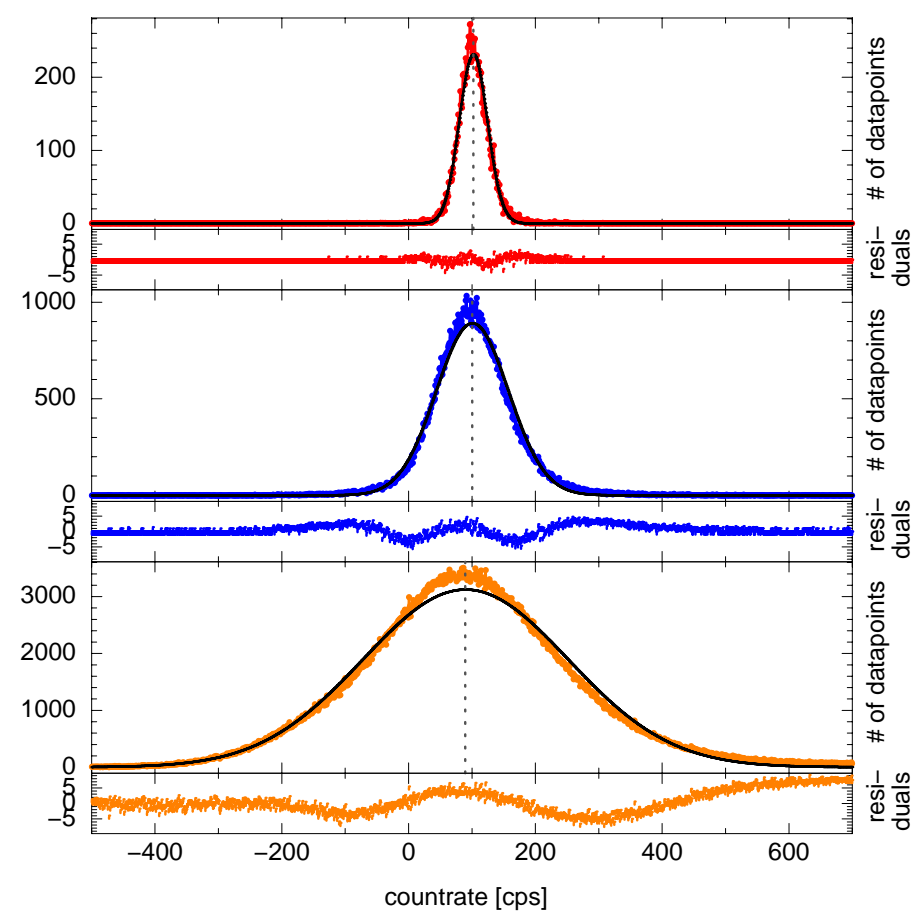

Fig. 3: Histograms (width $1 \mathrm{cps}$ ) of the ii_light lightcurves of revolution 628 with $10 \mathrm{~s}$ (upper panel), $1 \mathrm{~s}$ (middle panel) and $0.1 \mathrm{~s}$ (lower panel) time resolution and up to $\sim 15^{\circ}$ pointing offset angle in the $20-40 \mathrm{keV}$ band of Cyg X-1 and Gaussian fits to them. The dotted lines indicate the centers of the Gaussians

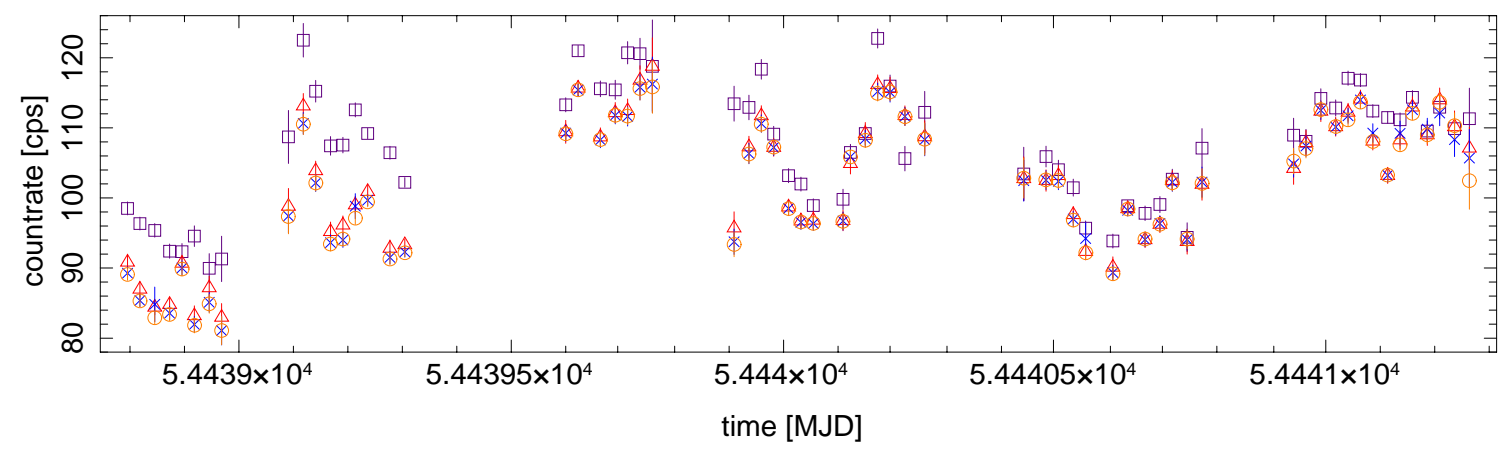

Fig. 4: Fluxes from image extraction (box; deconvolution algorithm consistent with ii_lc_extract) as well as averaged countrates for individual ScW of the ii_light lightcurves with $10 \mathrm{~s}$ (triangle) $1 \mathrm{~s}(\mathrm{x})$ and $0.1 \mathrm{~s}$ (circle) time resolution in the $20-40 \mathrm{keV}$ energy band

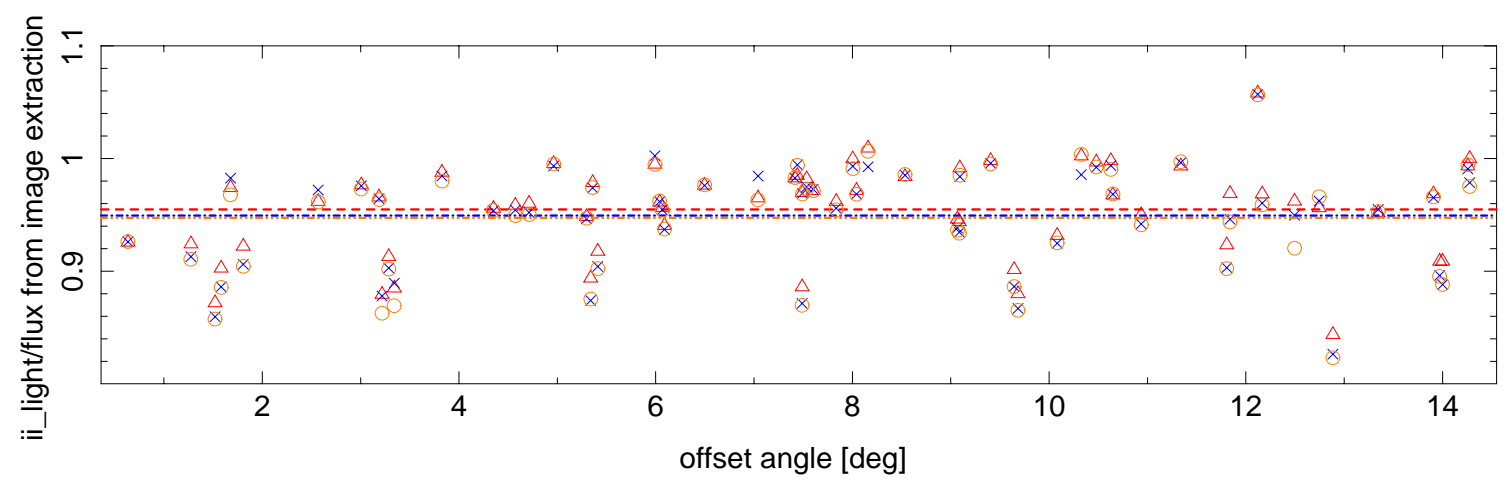

Fig. 5: The ratio between averaged countrates for individual ScWs of the ii_light lightcurves with 10s (triangle), (x) and $0.1 \mathrm{~s}$ (circle) time resolution in the $20-40 \mathrm{keV}$ energy band to the fluxes from the image extraction in dependency on the pointing offset angle of the science window 


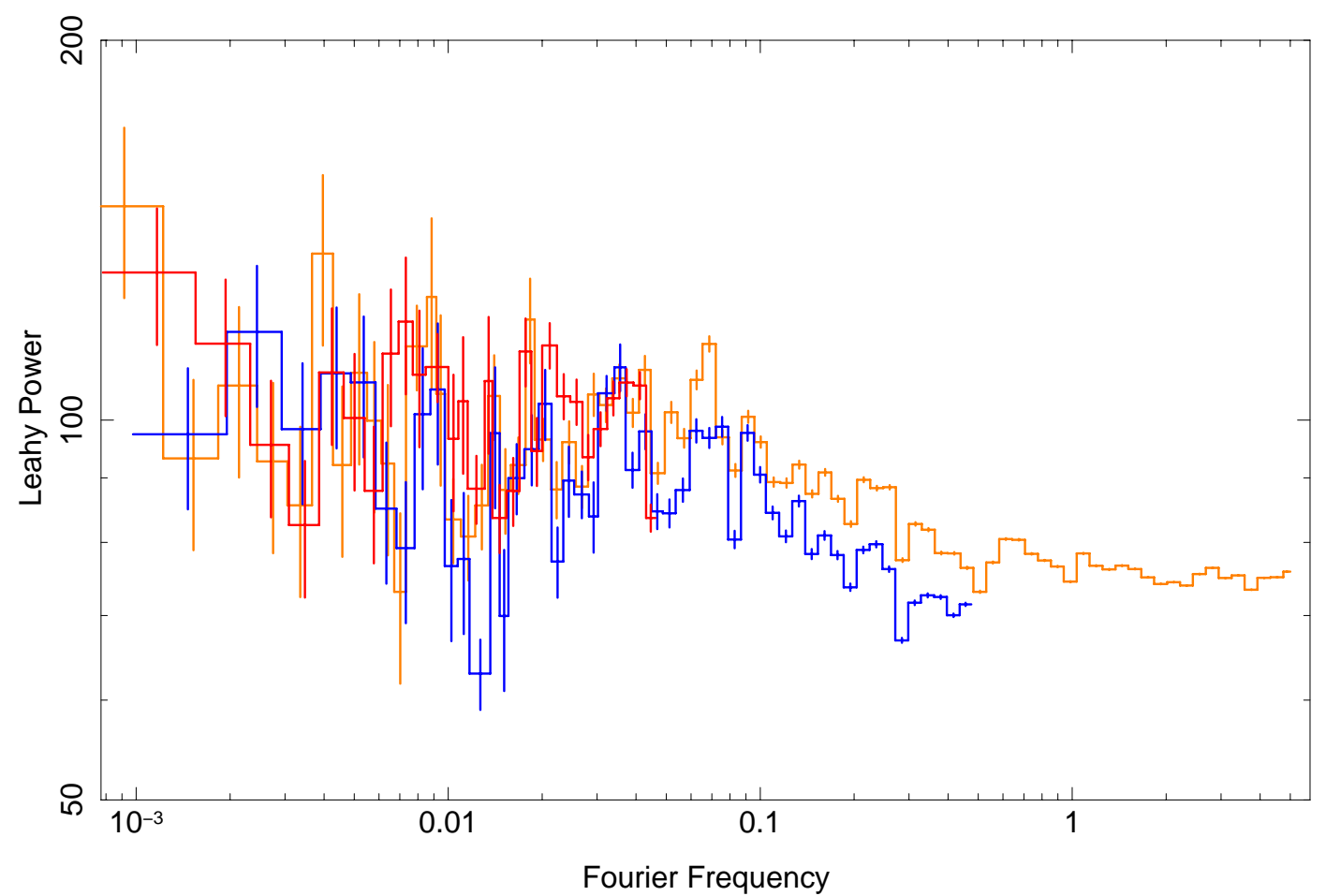

Fig. 6: Power spectrum densities (PSDs) for Cyg X-1 presented here for the lightcurves with 10s (red), 1s (blue) and $0.1 \mathrm{~s}$ (brown) time resolution in the $20-40 \mathrm{keV}$ energy band in the Leahy normalization

ing that ii_light underestimates the source flux the different time resolutions are, however, consistent among each other and reproduce the shape of the lightcurve well.

Comparing the ratio between averaged countrates for individual ScWs of the ii_light lightcurves to the fluxes from the image extraction, we see no offset angle dependency, as reported by [2], cf. Fig. 5. The respective means of the ratios (dotted lines) agree well and indicate an offset of $\sim 5 \%$, consistent with the linear correlation presented above for the fully coded FOV.

The power spectrum densities (PSDs) calculated from the above discussed ii_light lightcurves are shown in Fig. 6. For such PSDs calculated in Leahy normalizazion, the Poisson noise level should be equal to 2 , independent of the countrate of the source. It can, however, clearly be seen here that even at as high frequencies as a few $\mathrm{Hz}$, the PSD flattens out at a value of $\sim 80 \mathrm{rms}^{2} / \mathrm{Hz}$. This is consistent with the findings of [1] for Vela X-1, where the Poisson noise contributes as much as $100 \mathrm{rms}^{2} / \mathrm{Hz}$ at a given frequency.

Our PSDs for different time resolutions agree reasonably well with each other in shape (for exact timing studies longer periods than a single revolution would be necessary to reduce the uncertainities). Note that [3] also found consistent PSD shapes comparing ISGRI and RXTE-PCA $15-70 \mathrm{keV}$ data for
Cyg X-1. So while a better noise correction is required, ii_light lightcurves are still well suited for timing studies with a $10 \mathrm{~s}$ to $0.1 \mathrm{~s}$ resolution in the regime above $20 \mathrm{keV}$.

\section{Summary and Conclusions}

We have shown that it is possible to perform timing studies with a resolution of up to $0.1 \mathrm{~s}$ with $I N$ TEGRAL when using the ii_light tool. Although ii_light (OSA 7 version) systematically underestimates the countrates when compared to more exact deconvolution algorithms (which do not allow better time resolution than $10 \mathrm{~s}$ even for bright sources such as Cyg X-1), the differences can in principle be assessed and taken into account. The correlation between the countrates is linear, with the slope apparently depending on the field under consideration. A more detailed analysis of sources in different fields will allow to better quantify this linear correlation.

The countrates of the ii_light lightcurves follow a Gaussian distribution around the mean value. We do not see a dependency on the pointing offset angle of the observation.

PSDs calculated from these lightcurves with different time resolutions agree well with each other, the noise does however show anomalous behaviour which has also been observed by [1]. 


\section{References}

[1] Fürst, F., Kreykenbohm, I., Pottschmidt, K., et al.: $A \& \&$ \& $\mathbf{5 1 9}, 37,2010$.

[2] Kreykenbohm, I., Wilms, J., Kretschmar, P., et al.: $A \mathscr{E} A, \mathbf{4 9 2}, 511,2008$.

[3] Pottschmidt, K., Wilms, J., Nowak, M., et al.: Advances in Space Research, 38, 2006, p. 1350.

V. Grinberg

E-mail: Victoria.grinberg@sternwarte.uni-erlangen.de

Remeis-Observatory/ECAP/FAU

Sternwartstr. 7

D-960 49 Bamberg, Germany

USM/LMU, Munich, Germany

I. Kreykenbohm

Remeis-Observatory/ECAP/FAU

Bamberg, Germany

F. Fürst

Remeis-Observatory/ECAP/FAU

Bamberg, Germany

J. Wilms

Remeis-Observatory/ECAP/FAU

Bamberg, Germany
K. Pottschmidt

CRESST/NASA-GSFC

Greenbelt, MD, USA

UMBC, Baltimore, MD, USA

M. Cadolle Bel

ESAC, Madrid, Spain

J. Rodriguez

DSM/DAPNIA/SAp, CEA Saclay, France

D. M. Marcu

CRESST/NASA-GSFC

Greenbelt, MD, USA

UMBC, Baltimore, MD, USA

GMU, Fairfax, VA, USA

S. Suchy

CASS/UCSD, La Jolla, CA, USA

A. Markowitz

CASS/UCSD, La Jolla, CA, USA

M. A. Nowak

MIT/Chandra X-ray Center

Cambridge, MA, USA 